12

\title{
Генерация когерентного рентгеновского излучения гармоник в однопроходном лазере на свободных электронах со сдвигом фаз электронов относительно фотонов
}

\author{
(C) К.В. Жуковский, А.М. Калитенко \\ Московский государственный университет им. М.В. Ломоносова, \\ 119991 Москва, Россия \\ e-mail: zhukovsk@physics.msu.ru
}

Поступило в Редакцию 14 июля 2018 г.

В окончательной редакции 7 февраля 2020 г.

Принято к публикации 11 февраля 2020 г.

\begin{abstract}
Проведено теоретическое исследование эволюции банчинга (коэффициентов группировки) и мощности излучения гармоник в однопроходном лазере на свободных электронах (ЛСЭ), в котором осуществляется периодический сдвиг фаз между электронами и фотонами в промежутках между ондуляторами с гармониками магнитного поля. С помощью аналитического расчета выявлены конфигурации магнитного поля асимметричного эллиптического и плоского ондуляторов, которые позволяют несколько ослабить основной тон и усилить отдельные высшие гармоники. Для анализа эволюции мощности и коэффициентов группировки использована феноменологическая модель ЛСЭ, описывающая нарушение группировки между каскадами ондуляторов и повышенную чувствительность электрон-фотонного взаимодействия на длинах волн высших гармоник к разбросу энергии, размеру и отклонению пучка от оси и др. Аналитически исследованы выражения для коэффициентов Бесселя плоского и эллиптического ондуляторов с гармониками поля. Предложено применение этих ондуляторов в ЛСЭ со сдвигом фаз для увеличения мощности высших гармоник в рентгеновском диапазоне до 100 раз по сравнению с обычными ЛСЭ.
\end{abstract}

Ключевые слова: излучение ондуляторов, двухчастотный ондулятор, генерация гармоник, лазер на свободных электронах.

DOI: $10.21883 / J T F .2020 .08 .49545 .280-18$

\section{Введение}

Первые лазеры - приборы для генерации когерентного излучения - появились в 1960-х годах и работали в инфракрасном диапазоне. Обычные лазеры используют принцип инверсной заселенности рабочего тела; длина волны их излучения $\lambda$ ограничена снизу величиной $\sim 100 \mathrm{~nm}$. Для исследования процессов на наномасштабе требуются источники когерентного излучения в рентгеновском диапазоне, самым мощным из которых на протяжении многих лет было синхротронное излучение (СИ) [1]. Однако СИ, как и ондуляторное излучение (ОИ), не является когерентным. ОИ генерируется электронами в пространственнопериодическом магнитном поле, где на фоне релятивистского дрейфа вдоль оси ондулятора заряд совершает малые колебания поперек оси. Излучение, приходящее синфазно от каждого периода ондулятора, складывается на выходе в резонансы ОИ. Впервые идея об этом была высказана Гинзбургом [2] в середине XX века и воплощена Мотцом [3]; Гинзбург также выдвинул гипотезу о возможности получения когерентного ОИ от электронных банчей (сгустков), разделенных друг от друга длиной волны излучения; при этом длина банча должна быть меньше длины волны излучения. Эта идея была воплощена в лазерах на свободных электронах (ЛСЭ), где излучение взаимодействует с электронами в ондуляторе и группирует их вблизи узлов волны излучения [4-12]. ЛСЭ способны генерировать когерентное рентгеновское излучение с пиковой мощностью до гигаватта и более, что превосходит мощность СИ в рентгеновском диапазоне на несколько порядков. Рентгеновское излучение применяется во многих областях науки и техники, в медицине, архитектуре и др. (см, например, [13-18]). Ввиду отсутствия эффективных отражающих элементов в рентгеновском диапазоне для генерации в нем применяют ЛСЭ с высоким коэффициентом усиления [19-23], где излучение от электронов высокой энергии формируется за один проход излучения в ондуляторе большой длины. Для этого нужны пучки электронов с малым сечением, эмиттансом и малым разбросом энергии, большой ток (обычно килоамперы), и высокая плотность тока. Установки, излучающие в рентгеновском диапазоне, могут иметь размеры в километр и более, а суммарная длина ондуляторов в них достигает ста метров. С целью уменьшения размеров и стоимости рентгеновских ЛСЭ можно использовать генерацию гармоник [24-34]. Плоские и эллиптические двухчастотные ондуляторы генерируют гармоники ОИ в линейном режиме с бо́льшим коэффициентом усиления, чем обычные плоские ондуляторы [35-58]; это делает предпочтительным их использование в ЛСЭ в каскадах группирователей электронов [53-58]. 
Чтобы повысить мощность гармоник ЛСЭ, ограниченную насыщением основного тона, было предложено решение со сдвигом фаз между секциями ондуляторов [59,60]. Сдвиг микробанча электронов относительно волны излучения на $k \pi / n$, где $k=2,4,6$, не влияет на группировку электронов на длине волны $n$-й гармоники, но нарушает группировку на длине волны основного тона и других гармоник, смещая фактическое положение микробанчей относительно узлов волны поля излучения гармоник. Вышеописанные явления в обычных ондуляторах впервые изучались в $[59,60]$, а в двухчастотном плоском ондуляторе - в работах [61,62]. Отметим, что выражения для функций Бесселя в $[61,62]$ содержат опечатки, повлиявшие на моделирование мощности ЛСЭ; исправленные формулы для коэффициентов и обобщенных функций Бесселя приведены в $[51,54,63]$. Важным является вывод в $[59,60]$ о том, что можно ослабить основной тон ЛСЭ и повысить мощность 3-й гармоники излучения, используя расфазировку на каждой длине усиления ЛСЭ с обычным ондулятором. Заключение в отношении двухчастотного ондулятора в $[61,62]$ требует переработки ввиду ошибки в формулах функций Бесселя.

В настоящей работе нами исследуется излучение асимметричного эллиптического ондулятора с гармониками поля; выявляются параметры поля, позволяющие ослабить основной тон и усилить высшие гармоники излучения средствами самого ондулятора и обеспечить рост мощности гармоник ЛСЭ до их естественного насыщения. Для анализа эволюции мощности гармоник и банчинга в ЛСЭ мы используем феноменологическую модель [38,43,64], откалиброванную по экспериментам на ЛСЭ. Модификация модели для эллиптических ондуляторов с возможным сдвигом фаз между каскадами ондуляторов дана в [43]. С ее помощью нами аналитически исследуется генерация гармоник в ЛСЭ со сдвигом фаз электронов и фотонов между ондуляторами с гармониками поля, и выявляются преимущества использования в ЛСЭ эллиптического ондулятора с гармониками поля по сравнению с плоским ондулятором.

\section{1. Использование гармоник поля ондулятора для усиления гармоник ОИ}

\section{1. Плоский ондулятор с гармониками магнитного поля}

В работах [35-58] было показано, что двухчастотные ондуляторы могут генерировать излучение гармоник ОИ повышенной мощности по сравнению с обычным ондулятором. Для этого можно использовать, например, следующее двоякопериодическое магнитное поле с амплитудами $H_{0}$ и $d H_{0}$ :

$$
\begin{gathered}
\mathbf{H}=\left(0, H_{0}\left(\sin \left(k_{\lambda} z\right)+d \sin \left(h k_{\lambda} z\right)\right), 0\right), \\
k_{\lambda}=2 \pi / \lambda_{u}, h \in Z, d, h=\mathrm{const},
\end{gathered}
$$

где $\lambda_{u}$ - период основного периодического поля ондулятора. Резонансы ОИ возникают на следующих длинах волн:

$$
\lambda_{n}=\frac{\lambda_{u}}{2 n \gamma^{2}}\left(1+\left(k_{\mathrm{eff}}^{2} / 2\right)+(\gamma \theta)^{2}\right)
$$

где $k_{\mathrm{eff}}^{2}=k^{2}\left(1+(d / h)^{2}\right)$ - эффективный параметр ондулятора, $k=H_{0} \lambda_{u ; x} e / 2 \pi m_{e} c^{2}, e-$ заряд электрона, $m_{e}$ - масса электрона, $\gamma$ - релятивистский параметр электрона и $\theta-$ угол отклонения от оси ондулятора; для обычного плоского ондулятора $d=0$. Интенсивность ОИ, как обычно, определяется следующей формулой:

$$
\frac{d^{2} I}{d \omega d \Omega}=\frac{e^{2} N^{2} \gamma^{2} k^{2} \sum_{n=-\infty}^{\infty} n^{2} \operatorname{sinc}^{2}\left(v_{n} / 2\right)\left(\left|f_{n, x}\right|^{2}+\left|f_{n, y}\right|^{2}\right)}{c\left(1+\left(k_{\mathrm{eff}}^{2} / 2\right)+(\gamma \theta)^{2}\right)^{2}}
$$

где $n$ - номер гармоники ОИ, $N-$ число периодов ондулятора, $v_{n}=2 \pi n N\left(\left(\lambda_{n} / \lambda\right)-1\right)$ - параметр расстройки, $\lambda$ - длина волны излучения, $f_{n, x, y}-$ коэффициенты Бесселя для $x$ - и $y$-поляризаций. В зависимости от конфигурации поля ондулятора меняются резонансы ОИ (2) и коэффициенты Бесселя $f_{n, x, y}$. Для плоского ондулятора с полем (1) коэффициенты Бесселя зависят от обобщенных функций Бесселя $I_{n}^{m}$ следующим образом:

$$
\begin{gathered}
f_{\begin{array}{c}
\text { 2-frequency } \\
\text { planar und. }
\end{array}}^{f, x}=I_{n-1}^{n}+I_{n+1}^{n}+\frac{d}{h}\left(I_{n+h}^{n}+I_{n-h}^{n}\right)+I_{n}^{n} \frac{2}{k} \gamma \theta \cos \varphi, \\
\qquad \begin{array}{c}
n, x \\
\begin{array}{c}
n-f \text { frequency } \\
\text { planar und. }
\end{array}
\end{array}
\end{gathered}
$$

где $\theta$ - угол отклонения от оси ондулятора, $\varphi-$ азимутальный угол вокруг оси, а функции Бесселя $I_{n}^{m}$ имеют следующее интегральное представление [50,51]:

$$
\begin{gathered}
I_{n}^{m}=\int_{0}^{2 \pi} \frac{d \alpha}{2 \pi} \cos [n \alpha \\
\left.+\frac{m k^{2}\left(\xi_{0}(\alpha)+\xi_{1}(\alpha)+\xi_{2}(\alpha)+\xi_{3}(\alpha)+\xi_{4}(\alpha)+\xi_{5}(\alpha)\right)}{1+\gamma^{2} \theta^{2}+k_{\mathrm{eff}}^{2} / 2}\right] \\
\xi_{0}=\frac{2 d \gamma \theta \cos \varphi \sin (h \alpha)}{k h^{2}}, \quad \xi_{1}=\frac{\sin (2 \alpha)}{4} \\
\xi_{2}=\frac{d \sin ((h-1) \alpha)}{h(h-1)}, \xi_{3}=\frac{d \sin ((h+1) \alpha)}{h(h+1)} \\
\xi_{4}=\frac{d^{2} \sin (2 h \alpha)}{4 h^{3}}, \quad \xi_{5}=\frac{2}{k} \gamma \theta \cos \varphi \sin \alpha
\end{gathered}
$$

Формулы (4) и (5) исправляют опечатки в $[45,49,57,58,62,65,66]$ и добавляют важную аналитическую зависимость $f_{n, x, y}$ от угла отклонения от оси ондулятора.

В работе [44] была решена обратная задача по нахождению распределения поля ондулятора с линейно поляризованным излучением на оси в оптимальных условиях, 
и был сделан вывод, что поле такого ондулятора в основном описывается суммой его 1-й и 3-й гармоник. В $[38,42,43]$ независимо от [44] было показано, что выбор амплитуды $d \cong+0.53$-й гармоники поля, $h=3$, в (1) позволяет усилить 3-ю и 5-ю гармоники ОИ и несколько ослабить основной тон. Таким образом, ондулятор с полем (1) $d \cong+0.5, h=3$ можно использовать для усиления излучения гармоник в ЛСЭ в дополнение к предложенному в $[59,60]$ способу подавления основного тона и увеличения мощности гармоник излучения расфазировкой. Проведенные на эту тему последние исследования для двухчастотных ондуляторов [62,63] концептуально верны, но из-за наличия опечаток в коэффициентах Бесселя нужно пересмотреть поведение гармоник в ЛСЭ, что мы сделаем ниже в разд. 2.

\section{2. Эллиптический ондулятор с гармониками магнитного поля}

Спиральные и эллиптические ондуляторы в последнее время находятся в центре внимания исследователей, так как они позволяют эффективно выделять некоторые гармоники спектра ОИ. Простейшим примером служит спиральный ондулятор с синусоидальным полем; на его оси в идеале излучается только 1-я гармоника ОИ. Двухцветное лазерное излучение в ЛСЭ с эллиптическим ондулятором со скрещенными магнитными полями изучалось в [46]. Из-за сложности возникающих математических выражений для функций Бесселя, исследования эллиптических ондуляторов часто проводятся численно (см. $[39,40,41])$. Аналитическое исследование различных эллиптических ондуляторов с гармониками поля было проведено в [38,42,43], где были получены выражения для их коэффициентов Бесселя. Там же было показано, что спиральный ондулятор с антисимметричной 3-й гармоникой поля

$$
\begin{gathered}
H=H_{0}\left(\sin \left(k_{\lambda} z\right)-d \sin \left(h k_{\lambda} z\right), \cos \left(k_{\lambda} z\right)+d \cos \left(h k_{\lambda} z\right), 0\right), \\
h \in \text { integer, }
\end{gathered}
$$

при $d=0.5$ излучает заметную 5-ю гармонику ОИ на фоне мощного основного тона, а остальные гармоники слабы. Однако 5-я гармоника излучения практически полностью индуцирована основным тоном ЛСЭ в режиме нелинейной генерации, что не позволяет получить мощность 5-й гармоники выше мощности основного тона.

В этом отношении представляет интерес асимметричный эллиптический ондулятор с 3-й гармоникой поля по одной координате. Ниже мы проведем теоретическое исследование генерации спонтанного излучения в таком ондуляторе, а в разд. 2 исследуем возможность получить мощную 3-ю гармонику вынужденного излучения в ЛСЭ с таким ондулятором, и сравним его с ЛСЭ с обычным ондулятором.
Рассмотрим следующее троякопериодическое эллиптическое магнитное поле:

$$
\begin{gathered}
\mathbf{H}=H_{0}\left(\sin \left(k_{\lambda} z\right), d_{1} \sin \left(h k_{\lambda} z\right)+d_{2} \cos \left(l k_{\lambda} z\right), 0\right), \\
l \neq h, \quad l, h \in \text { integer, }
\end{gathered}
$$

с кратными периодами ондулятора $\lambda_{u ; x} \equiv \lambda_{u}, \lambda_{u ; y}^{1}=\lambda_{u} / h$, $\lambda_{u ; y}^{2}=\lambda_{u} / l, \quad k_{\lambda}=2 \pi / \lambda_{u} . \quad$ В предельных случаях $d_{1}=0 \cap d_{2} \neq 0 \quad$ и $\quad d_{2}=0 \cap d_{1} \neq 0, \quad$ формула $\quad$ (7) описывает эллиптические ондуляторы со скрещенным полем. При $d_{2}=0 \cap d_{1}=h=1$ (7) описывает плоский ондулятор с поляризацией излучения в плоскости под углом $45^{\circ}$ к взаимно-ортогональным магнитным полям, и $k_{\mathrm{eff}}=\sqrt{2} k$. При $d_{2}=l=1 \cap d_{1} \neq 0$ получаем поле спирального ондулятора с гармоникой в одной плоскости. При $d_{2}=l=1 \cap d_{1}=0$ поле (7) описывает обычный спиральный ондулятор. Вычисления интеграла излучения для электрона в поле (7) проводятся стандартным образом: в экспоненте в интеграле выделяются периодическая и непериодическая части; нули фазы этой экспоненты дают резонансы ОИ на длинах волн $\lambda_{n}$, которые для кратных периодов ондулятора и соответственно кратных $l$ и $h$ в (7) записываются под углом $\theta$ к оси в виде (2), где $\quad k_{\mathrm{eff}}^{2}=k^{2}\left(1+\left(d_{1} / h\right)^{2}+\left(d_{2} / l\right)^{2}\right) . \quad$ Вычисление периодической части показателя экспоненты интеграла излучения для кратных периодов ондулятора с полем (7) и $l \neq h$ приводит к следующим обобщенным функциям Бесселя в интегральном представлении:

$$
\begin{gathered}
J_{n}^{m}=\int_{0}^{2 \pi} \frac{d \alpha}{2 \pi} \\
\times \cos \left(n \alpha+\frac{m k^{2}\left(\xi_{0}+\xi_{1}+\xi_{2}+\xi_{3}+\xi_{5}+\xi_{6}+\xi_{7}+\xi_{8}\right)}{1+\left(k_{\mathrm{eff}}^{2} / 2\right)+\gamma^{2} \theta^{2}}\right), \\
\xi_{1}=\frac{\sin (2 \alpha)}{4}, \quad \xi_{0}=\frac{2 d_{1} \sin (h \alpha)}{k h^{2}} \gamma \theta \cos \varphi \\
\xi_{2}=\frac{2 d_{2} \cos (l \alpha)}{k l^{2}} \gamma \theta \cos \varphi, \xi_{3}=\frac{2 \sin \alpha}{k} \gamma \theta \sin \varphi \\
\xi_{5}=\frac{d_{1}^{2} \sin (2 h \alpha)}{4 h^{3}}, \quad \xi_{6}=-\frac{d_{2}^{2} \sin (2 l \alpha)}{4 l^{3}}, \\
\xi_{7}=\frac{d_{1} d_{2} \cos ((l+h) \alpha)}{h l(l+h)}, \quad \xi_{8}=\frac{d_{1} d_{2} \cos ((l-h) \alpha)}{h l(l-h)} .
\end{gathered}
$$

Соответствующие коэффициенты Бесселя $f_{n, x, y}$ записываются следующим образом:

$$
\begin{aligned}
\underbrace{f}_{\begin{array}{c}
\text { asymmetric } \\
\text { elliptic und. }
\end{array}}=\mid \frac{d_{1}}{h}\left(J_{n+h}^{n}+J_{n-h}^{n}\right) & +i \frac{d_{2}}{l}\left(J_{n+l}^{n}-J_{n-l}^{n}\right) \\
& +\frac{2}{k} \gamma \theta \cos \varphi J_{n}^{n} \mid, \\
\underbrace{f, y}_{\begin{array}{c}
\text { asymmetric } \\
\text { elliptic und. }
\end{array}} & =\left|J_{n+1}^{n}+J_{n-1}^{n}+\frac{2}{k} \gamma \theta \sin \varphi J_{n}^{n}\right|,
\end{aligned}
$$

где $J_{n}^{m}$ определена в (8). Полная интенсивность ОИ определяется выражением (3) с 


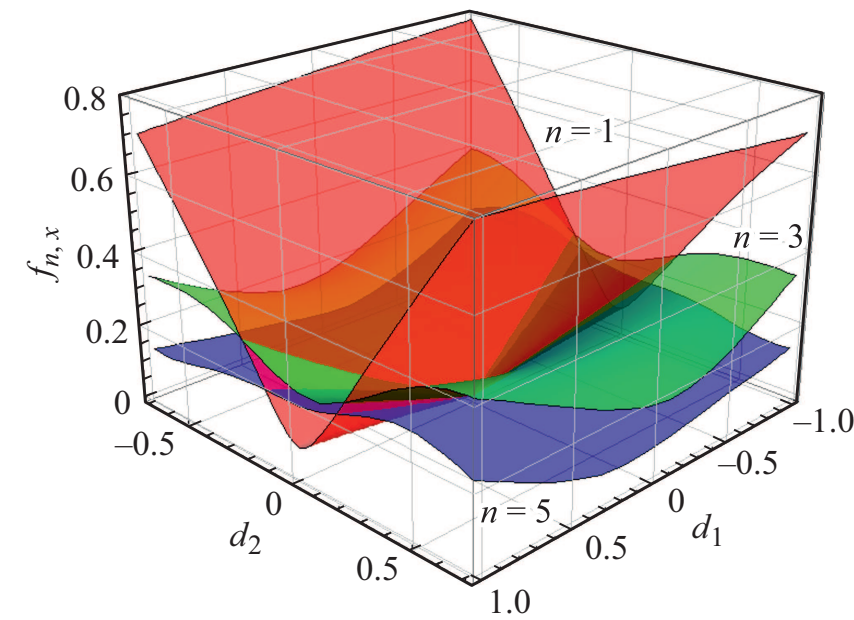

Рис. 1. Коэффициенты Бесселя для гармоник $x$-поляризации в ондуляторе с полем (7) с параметрами $k=3.5, h=3, l=1$; параметры электронного пучка: $\gamma=8400, \varepsilon^{n}=0.56 \mathrm{~mm} \cdot \mathrm{mrad}$, $\beta=15 \mathrm{~m}$

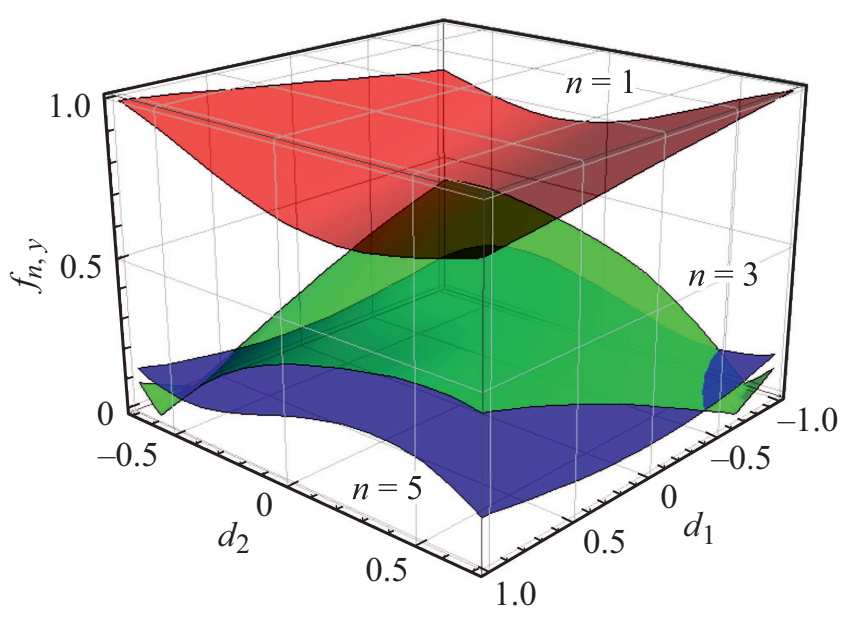

Рис. 2. Коэффициенты Бесселя для гармоник $y$-поляризации в ондуляторе с полем (7) с параметрами $k=3.5, h=3, l=1$; параметры электронного пучка: $\gamma=8400, \varepsilon^{n}=0.56 \mathrm{~mm} \cdot \mathrm{mrad}$, $\beta=15 \mathrm{~m}$.

$k_{\text {eff }}^{2}=k^{2}\left(1+\left(d_{1} / h\right)^{2}+\left(d_{2} / l\right)^{2}\right)$. В реальных устройствах угловое отклонение от оси $\gamma \theta$ имеет порядок $10^{-2}$, так что членом $(\gamma \theta)^{2}$ в большинстве случаев можно пренебречь по сравнению с единицей.

Зависимость коэффициентов Бесселя $f_{n, x, y}$, определяющих интенсивности гармоник излучения, от параметров $d_{1,2}$ поля ондулятора показана на рис. 1 для $x$-поляризации и на рис. 2 для $y$-поляризации. Гармоникам ОИ с $n=1,3,5$ соответствуют поверхности, обозначенные цифрами $1,3,5$. На рис. 1 видно, что большие коэффициенты Бесселя высших гармоник и соответственно большая интенсивность их излучения получаются при больших значениях $d_{1} \cong 1$ и при значениях $d_{2} \sim 0.5-0.7$ для $n=3$ и $d_{2} \sim 0.2-0.5$ для $n=5$. Более того, при $h=3, l=1, d_{1}=1, d_{2}=0.2-0.4$, имеем примерно равные друг другу значения коэффициентов Бесселя для 1-й, 3-й и 5-й гармоник в $x$ поляризации излучения, $f_{1 ; x} \simeq f_{3 ; x} \simeq f_{5 ; x}$ и наименьшие значения для коэффициента Бесселя основного тона $f_{1 ; y}$ для $y$-поляризации.

Поэтому ондулятор с полем (7), где $h=3, l=1$, $d=0, d_{1}=1, d_{2}=0.2-0.4$, может быть использован в ЛСЭ со сдвигом фазы между каскадами для более эффективного подавления основного тона и усиления высших гармоник когерентного излучения. Моделирование излучения ЛСЭ с различными двухчастотными ондуляторами будет представлено в следующем разделе. В частности, мы проведем моделирование ЛСЭ с асимметричным эллиптическим ондулятором с полем (7) и сравним ЛСЭ с плоским ондулятором.

\section{2. Излучение однопроходных ЛСЭ с расфазировкой между каскадами}

\section{1. ЛСЭ с асимметричным эллиптическим ондулятором со сдвигом фаз}

Несмотря на то, что двухчастотный ондулятор может улучшить генерацию высших гармоник по сравнению с обычным ондулятором, экспоненциальный рост мощности гармоник в нем по-прежнему ограничен насыщением основного тона, который индуцирует значительный разброс энергии электронов. Это приводит к тому, что высшие гармоники ЛСЭ насыщаются на длине насыщения основного тона $L_{g}$ или раньше $[29,59,67,68]$. Чтобы получить большие мощности высших гармоник, они должны расти далее по длине ондуляторов, так как естественное насыщение гармоник достигается за длиной насыщения $L_{g}$ основного тона. Это может быть достигнуто путем фильтрации основной гармоники или введения фазового сдвига $k \pi / n$, где $k=2,4,6$, электронов относительно фотонов между каскадами ЛСЭ (см. $[59,60])$. Сдвиг электронов относительно фотонов на $k \pi / n$ между каскадами смещает фактическое положение микробанчей относительно узлов волны поля излучения и нарушает группировку электронов на длине волны основного тона и низших по отношению в $\mathrm{n}$ гармоник. В то же время сдвиг на $k \pi / n$, где $k=2,4,6$ и т.д., не влияет на группировку на длине волны $n$-й гармоники и не подавляет ее генерацию. Так, можно выделить $n$-ю гармонику и ее высшие субгармоники и одновременно ослабить другие гармоники. Моделирование описанного выше ЛСЭ с плоским ондулятором $[59,60]$ показало, что для эффективного подавления основного тона излучения необходимо нарушение группировки электронов на каждой длине усиления. Ниже мы исследуем излучение ЛСЭ с разгруппировкой электронов между каскадами двухчастотных эллиптического и плоского ондуляторов и сравним с плоским ондулятором. Для этого мы используем феноменологическую модель [43], в которой учитываются все основные потери и их зависимости от гармоник излучения в ЛСЭ. 
Таблица 1. Данные моделирования ЛСЭ с асимметричным эллиптическим ондулятором

\begin{tabular}{l|c|c|c}
\hline Номер гармоники & $n=1$ & $n=3$ & $n=5$ \\
\hline Коэффициент Бесселя $f_{n} ; x$ & 0.47 & 0.33 & 0.26 \\
\hline Коэффициент Бесселя $f_{n} ; y$ & 0.77 & 0.43 & 0.24 \\
\hline Параметр Пирса $\rho_{n ; x}$ & 0.0006 & 0.0005 & 0.0005 \\
\hline Параметр Пирса $\rho_{n ; y}$ & 0.0009 & 0.0007 & 0.0004 \\
\hline Длина волны гармоники ЛСЭ $\lambda_{n}, \mathrm{~nm}$ & 1.75 & 0.58 & 0.35 \\
\hline $\begin{array}{l}\text { Мощность насыщения гармоник } \\
\text { в ЛСЭ с фазовым сдвигом } P_{F, n}, \mathrm{~W}\end{array}$ & $1 \cdot 10^{9}$ & $7 \cdot 10^{9}$ & $8 \cdot 10^{6}$ \\
\hline $\begin{array}{l}\text { Мощность насыщения гармоник } \\
\text { в ЛСЭ без фазового сдвига } P_{F, n}, \mathrm{~W}\end{array}$ & $9 \cdot 10^{9}$ & $2 \cdot 10^{8}$ & $1.5 \cdot 10^{7}$
\end{tabular}

Примечание. Параметры пучка электронов: релятивистский фактор электронов $\gamma=8400$, мощность пучка $P_{E}=4.3 \mathrm{TW}$, электронный ток $I_{0}=1 \mathrm{kA}$, плотность тока $J=1.573 \cdot 10^{11} \mathrm{~A} / \mathrm{m}^{2}$, сечение пучка $\Sigma_{\text {beam }}=2 \pi \varepsilon \beta=6.36 \cdot 10^{-9} \mathrm{~m}^{2}$, средний радиус пучка $r=\sqrt{\Sigma_{\text {beam }} / \pi}=45 \mu \mathrm{m}$, средний размер пучка $\sigma_{x, y}=\sqrt{\varepsilon \beta} \approx 30 \mu \mathrm{m}$, эмиттанс $\varepsilon_{x, y}^{n}=\gamma \varepsilon_{x, y}=0.56 \mathrm{~mm} \cdot \mathrm{mrad}$, параметр Твисса $\beta=15 \mathrm{~m}$, расходимость $\theta=\sqrt{\varepsilon / \beta} \approx 2 \mu \mathrm{rad}$, разброс энергий $\sigma_{e}=10^{-4}$.

Параметры ондулятора в (7): $k=3.5, \quad h=3, \quad l=1, \quad d_{1}=1$, $d_{2}=+0.4, \lambda_{u}=2.8 \mathrm{~cm}$.

Параметры ЛСЭ: параметр Пирса $\tilde{\rho}_{n} \cong(7-9) \cdot 10^{-4}$, длина насыщения 3-й гармоники с фазовым сдвигом $L_{s 3 ; x} \cong 33 \mathrm{~m}, L_{s 3 ; y} \cong 28 \mathrm{~m}$, длина усиления $L_{\text {gain } ; x}=2 \mathrm{~m}, L_{\text {gain } ; y}=1.5 \mathrm{~m}$, средний размер фотонного пучка $\sigma_{\text {photon }} \approx \sqrt{\sigma_{x, y} \sqrt{\lambda_{1} L_{g} / 4 \pi}} \cong 20 \mu \mathrm{m}$.

Рассмотрим многокаскадный ЛСЭ с асимметричным эллиптическим ондулятором с полем (7), где $h=3$, $l=1, d_{1}=1, d_{2}=0.4$; параметры пучка электронов, главный период ондулятора и его параметр $k$ выберем такими же, как в эксперименте LCLS $[69,70]$ (табл. 1). В соответствии с результатами разд. 1 в рассматриваемом нами ондуляторе может быть усилена 3-я гармоника излучения. Соответствующий сдвиг фаз электронов и фотонов на $k \pi / 3, k=2,4,6 \ldots$, между каскадами не влияет на независимую группировку 3-й гармоники ЛСЭ; микробанчи оказываются сдвинутыми относительно фотонов на длине волны излучения гармоник с $n=1,5,7$, и каждый раз происходит перегруппировка электронов относительно волн излучения $\lambda_{1}, \lambda_{5}$. С выбранными значениями параметров (табл. 1) получаем следующие длины волн и полные мощности гармоник ЛСЭ: для основного тона $\lambda_{1}=1.7 \mathrm{~nm}, P_{\lambda=1.7 \mathrm{~nm}}=1 \mathrm{GW}$, для третьей гармоники $\lambda_{3}=0.6 \mathrm{~nm}$ и $P_{\lambda=0.6 \mathrm{~nm}}=7 \mathrm{GW}$. Эволюция банчинга и мощности гармоник ЛСЭ показаны на рис. 3,4 для $\mathrm{X}$-поляризации и на рис. 5,6 для Y-поляризации излучения. Независимый рост мощности пятой гармоники на $\lambda_{5}=0.3 \mathrm{~nm}$ идет слабо из-за расфазировки ЛСЭ; индуцированный рост ее мощности за счет 1-й гармоники происходит в последней секции ЛСЭ. Доминирующая 3-я гармоника ЛСЭ индуцирует субгармоники на длинах волн $\lambda_{3 \times 3}=0.2 \mathrm{~nm}$ и $\lambda_{3 \times 5}=0.12 \mathrm{~nm}$.

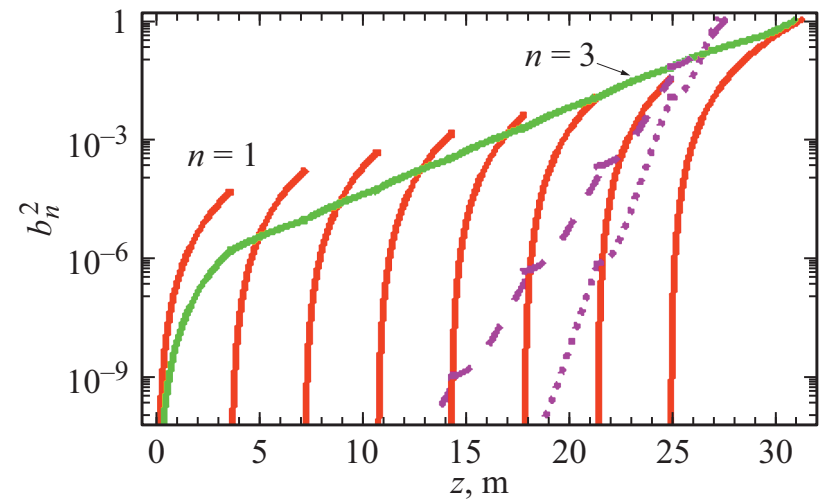

Рис. 3. Х-поляризация: коэффициенты банчинга в ЛСЭ со сдвигом фаз между каскадами эллиптического ондулятора с полем (7), где $h=3, l=1, d_{1}=1, d_{2}=0.4$. Номер гармоники $n=1, n=3$ отмечен на рисунке; субгармоники 3-й гармоники излучения: $n=3 \times 3$ - штриховая линия, $n=5 \times 3$ - пунктирная линия. Банчинг слабой 5-й гармоники не показан, чтобы не загромождать рисунок.

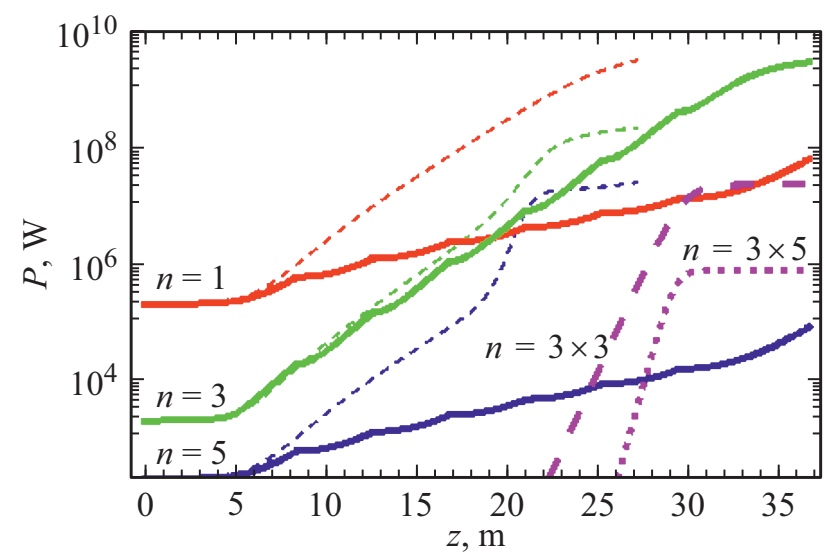

Рис. 4. Х-поляризация: мощность гармоник в ЛСЭ со сдвигом фаз между каскадами эллиптического ондулятора с полем (7), где $h=3, l=1, d_{1}=1, d_{2}=0.4$. Номера гармоник $n=1,3,5$ отмечены на рисунке; субгармоники 3-й гармоники излучения: $n=3 \times 3$ - жирная штриховая линия, $n=5 \times 3-$ жирная пунктирная линия.

Некоторая асимметрия характеристик излучения по поляризациям вызвана асимметрией поля ондулятора. Отметим, что при $d_{1}=d_{2}=0$ получаем поле ондулятора в эксперименте ЛCЭ LCLS, а параметры пучка при моделировании мы взяли из этого же эксперимента.

На рис. 4 и 6 видно, что суммарная мощность насыщения 3-й гармоники превышает мощность основного тона. Тонкими штриховыми линиями на правых графиках на рис. 4 и 6 показаны для сравнения мощности гармоник ЛСЭ с таким же эллиптическим ондулятором, но без сдвига фаз между каскадами; мощность 3-й гармоники оказываются на 1-2 порядка меньше (ср. тонкую штриховую линию с толстой сплошной линией для $n=3)$. Сравним эти результаты с результатами экспе- 


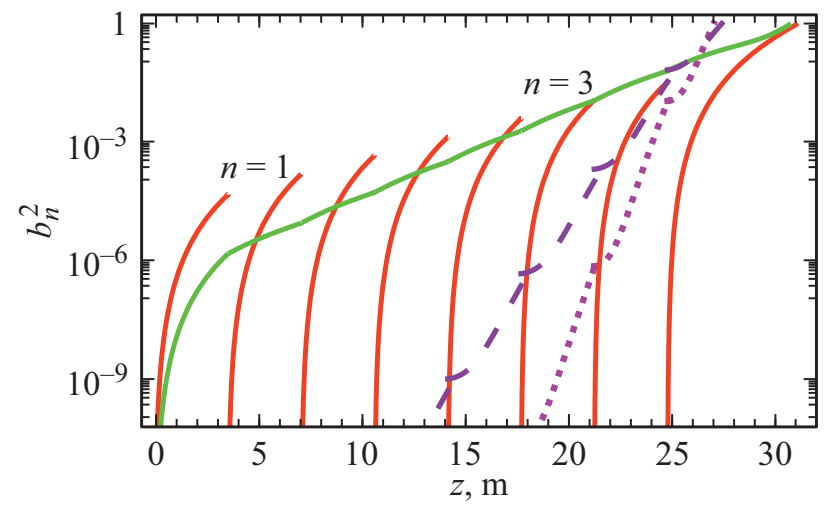

Рис. 5. Ү-поляризация: коэффициенты банчинга в ЛСЭ со сдвигом фаз между каскадами эллиптического ондулятора с полем (7), где $h=3, l=1, d_{1}=1, d_{2}=0.4$. Номера гармоник $n=1,3$ отмечены на рисунке; субгармоники 3 -й гармоники излучения: $n=3 \times 3$ - штриховая линия, $n=5 \times 3$ - пунктирная линия. Банчинг слабой 5-й гармоники не показан, чтобы не загромождать рисунок.

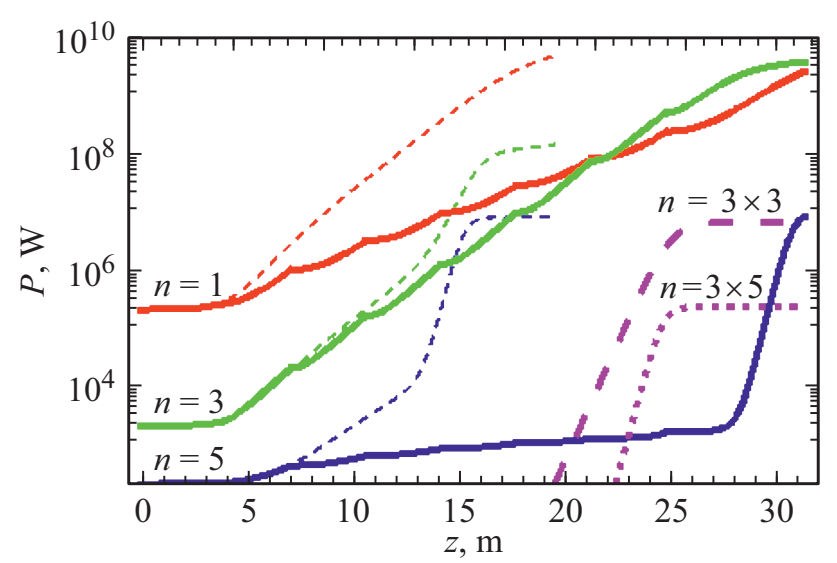

Рис. 6. Ү-поляризация: мощность гармоник в ЛСЭ со сдвигом фаз между каскадами эллиптического ондулятора с полем (7), где $h=3, l=1, d_{1}=1, d_{2}=0.4$. Номера гармоник $n=1,3,5$ и субгармоник отмечены на рисунке ; субгармоники 3-й гармоники излучения: $n=3 \times 3$ - штриховая линия, $n=5 \times 3-$ пунктирная линия.

римента ЛCЭ LCLS [69,70]. Длина волны основного тона в эксперименте при подобных условиях составляла $\lambda_{1}=1.5 \mathrm{~nm}$. При этом мощности гармоник ЛСЭ LCLS c использованном в эксперименте плоским ондулятором с полем (7) при $d_{1}=d_{2}=0$ без сдвига фаз составляли по данным [69,70]: $P_{\lambda 1=1.5 \mathrm{~nm}} \sim 5 \mathrm{GW}, P_{\lambda 3=0.5 \mathrm{~nm}} \sim 70 \mathrm{MW}$, $P_{\lambda 5=0.3 \mathrm{~nm}} \sim 7 \mathrm{MW}$. Сравнивая эти величины с результатами моделирования ЛСЭ с асимметричным эллиптическим ондулятором в табл. 1 и на рис. 4,6 , заключаем, что с предложенным ондулятором можно получить в $\sim 100$ раз большую мощность третьей гармоники на длине волны $\lambda_{3}=0.6 \mathrm{~nm}$, чем в эксперименте LCLS в подобных условиях. Кроме того, с предложенным ондулятором получаем излучение мощностью $\sim 30 \mathrm{MW}$ на длине волны третьей субгармоники $\lambda_{3 \times 3}=0.2 \mathrm{~nm}$, что в несколько раз выше мощности пятой гармоники LCLS на более длинной волне, $\lambda_{5}=0.3 \mathrm{~nm}$. Отметим, что в рентгеновском диапазоне эмиттанс пучка электронов может превышать длину волны излучения, деленную на $4 \pi$. Для 3-й гармоники, $n=3$, в эксперименте LCLS имеем $\varepsilon_{x, y} \approx \lambda_{3} / 4 \pi$; для $n>3: \varepsilon_{x, y}>\lambda_{3} / 4 \pi$.

На графиках на рис. 3,5 показаны коэфициенты банчинга основного тона $n=1$, 3-й гармоники $n=3$ и eе 3-й (штриховая линия) и 5-й (пунктирная линия) субгармоник $n=3 \times 3$ и $n=5 \times 3$ соответственно. На длине волны 3-й гармоники ОИ и ее субгармоник рост мощности и банчинга проходит практически монотонно и непрерывно, в то время как на длине волны основного тона $n=1$ разгруппировка электронов между каскадами приводит к разрывам банчинга (см. разрывную линию). Перегруппировка электронов после каждого сдвига банча электронов относительно фотонов происходит достаточно быстро, банчинг восстанавливается и растет до следующего разрыва между каскадами, как видно на рис. 3, 5. Рост мощности излучения следует за эволюцией банчинга; для 1-й и 5-й гармоник он идет медленнее, чем для 3-й гармоники (ср. линии $n=1$ и $n=5$ с линией $n=3$ на рис. 4 и 6); 5-я гармоника в конце ЛСЭ индуцируется 1-й гармоникой в нелинейном режиме генерации. Не ограниченная насыщением основного тона, 3-я гармоника в конце ЛСЭ оказывается мощнее 1-й. Этому способствуют более высокие по сравнению с обычным плоским ондулятором значения коэффициентов Бесселя для 3-й гармоники и более низкие значения для коэффициентов Бесселя основного тона.

\section{2. ЛСЭ с плоским двухчастотным ондулятором со сдвигом фаз}

Для сравнения с результатами предыдущего раздела, ниже мы смоделировали ЛСЭ с двухчастотным плоским ондулятором с полем (1), где, следуя выводу разд. 1.1, полагаем для второго периодического поля ондулятора $h=3, d=+0.5$, чтобы несколько ослабить основной тон и усилить 3-ю гармонику ОИ. Число каскадов, сдвиги фаз, параметры пучка и параметры основного поля ондулятора остаются такими же, как в ЛСЭ с асимметричным эллиптическим ондулятором (7) (см. разд. 2.1). Отметим, что в предельном случае поля (1) с $d=0$, т.е. с единственным периодическим полем, получаем параметры обычного плоского ондулятора из эксперимента LCLS. Эволюция банчинга и мощности гармоник ЛСЭ вдоль оси двухчастотных ондуляторов с полем (1), где $h=3, d=+0.5$, показаны соответственно на рис. 7 и 8 . Основные параметры ЛСЭ с плоским двухчастотным ондулятором, отличные от параметров ЛСЭ с эллиптическим ондулятором, приведены в табл. 2.

В целом характер поведения мощности и коэффициентов группировки (банчинга) гармоник ЛСЭ с плоским двухчастотным ондулятором похож на их поведение в 


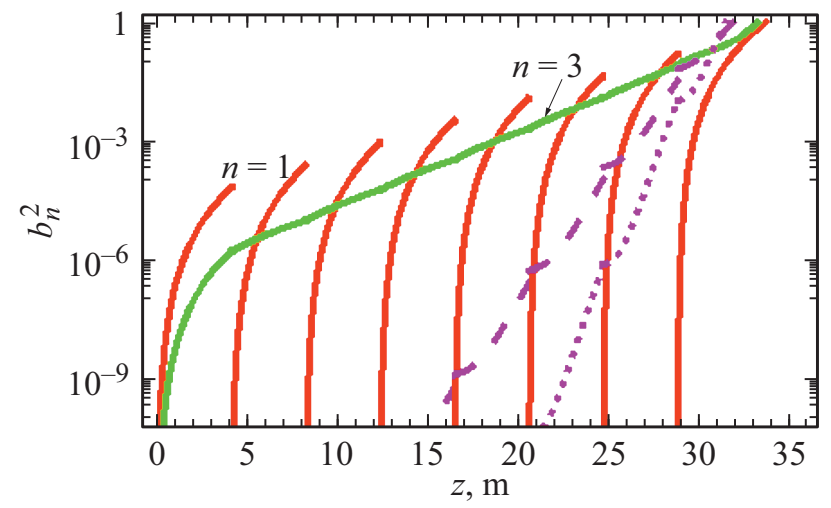

Рис. 7. Коэффициенты банчинга в ЛСЭ со сдвигом фаз между каскадами плоского двухчастотного ондулятора с полем (1), где $h=3, d=0.5$. Номера гармоник $n=1,3$ отмечены на рисунке; субгармоники 3-й гармоники излучения: $n=3 \times 3-$ штриховая линия, $n=5 \times 3-$ пунктирная линия.

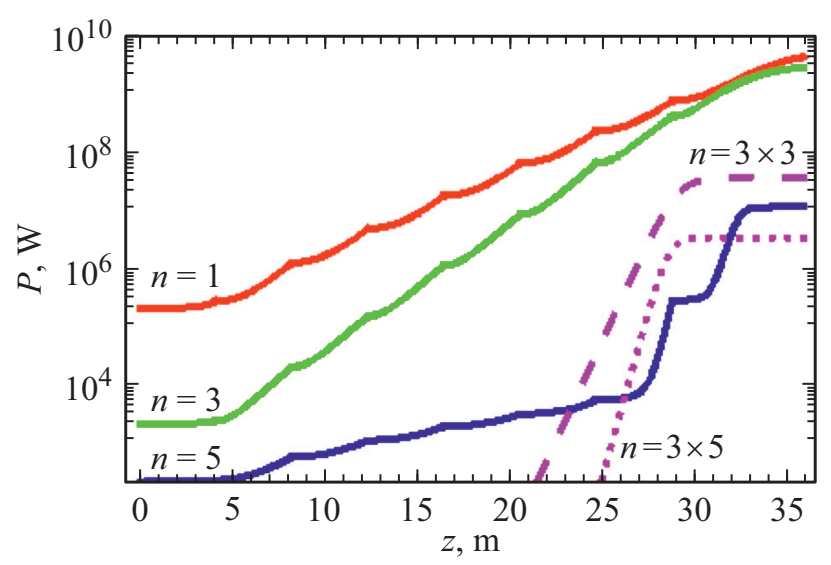

Рис. 8. Мощность гармоник в ЛСЭ со сдвигом фаз между каскадами плоского двухчастотного ондулятора с полем (1), где $h=3, d=0.5$. Номера гармоник $n=1,3$ и субгармоник $n=3 \times 3,5 \times 3$ отмечены на рисунке; субгармоники 3-й гармоники излучения: $n=3 \times 3-$ штриховая линия, $n=5 \times 3-$ пунктирная линия.

ЛСЭ с эллиптическим ондулятором, рассмотренном в разд. 2.1. Основное отличие состоит в том, что практически отсутствует вторая поляризация излучения плоского ондулятора на его оси. Также отметим, что в плоском двухчастотном ондуляторе несколько меньше коэффициенты Бесселя для 3-й и 5-й гармоник и больше для 1-й гармоники по сравнению с эллиптическим ондулятором (ср. с табл. 1). Поэтому подавление основного тона и усиление гармоник в ЛСЭ с плоским ондулятором происходит медленнее, чем в ЛСЭ с эллиптическим ондулятором.

Мощность 1-й и 3-й гармоник излучения примерно равны друг другу в конце ЛСЭ с плоскими двухчастотными ондуляторами (см. линии $n=1, n=3$ на длине $>30 \mathrm{~m}$ на рис. 8). Длины усиления и насыщения, частоты излучения и другие параметры близки к параметрам
Таблица 2. Данные моделирования ЛСЭ с двухчастотным плоским ондулятором

\begin{tabular}{l|c|c|c}
\hline Номер гармоники & $n=1$ & $n=3$ & $n=5$ \\
\hline Коэффициент Бесселя $f_{n ; y}$ & 0.69 & 0.35 & 0.24 \\
\hline Длина усиления гармоники $L_{\text {gain }}, \mathrm{m}$ & 1.6 & 1.9 & 2.2 \\
\hline Параметр Пирса $\rho_{n ; y}$ & 0.0009 & 0.0005 & 0.0004 \\
\hline Длина волны гармоники $\lambda_{n}, \mathrm{~nm}$ & 1.45 & 0.48 & 0.29 \\
\hline $\begin{array}{l}\text { Мощность насыщения гармоник } \\
\text { в ЛСЭ с фазовым сдвигом } P_{F, n}, \mathrm{~W}\end{array}$ & $5 \cdot 10^{9}$ & $3 \cdot 10^{9}$ & $1 \cdot 10^{7}$ \\
\hline $\begin{array}{l}\text { Мощность насыщения гармоник } \\
\text { в ЛСЭ без фазового сдвига } P_{F, n}, \mathrm{~W}\end{array}$ & $5 \cdot 10^{9}$ & $9 \cdot 10^{7}$ & $1 \cdot 10^{7}$
\end{tabular}

Пр имечан и е. Параметры пучка электронов: см. табл. 1; параметры ондулятора с полем (1): $k=3.5, h=3, d=+0.5, \lambda_{u}=2.8 \mathrm{~cm}$; параметры ЛСЭ: параметр Пирса $\tilde{\rho}_{n} \cong(4-9) \cdot 10^{-4}$, длина насыщения для ЛСЭ с фазовым сдвигом $L_{s 1} \cong L_{s 3} \cong 30 \mathrm{~m}$, длина насыщения без фазового сдвига $L_{s} \cong 20 \mathrm{~m}$.

ЛСЭ в разд. 2.1. Дальнейшее увеличение амплитуды второго периодического поля $d$ в (1) от значения 0.5 до 1 не значительно усиливает 3-ю гармонику ЛСЭ и не позволяет ей доминировать над 1-й гармоникой. Отметим, что мощный основной тон плоского ондулятора индуцирует 5-ю гармонику в нелинейном режиме (линия $n=5$ на рис. 8). Напротив, в ЛСЭ с асимметричными эллиптическими ондуляторами с тем же числом каскадов 5-я гармоника слабая, так как основной тон в таком ЛСЭ лучше подавлен и 3-я гармоника доминирует к концу ЛСЭ. Таким образом, асимметричный эллиптический ондулятор с 3-й гармоникой поля (7) позволяет более эффективно выделить 3-ю гармонику ЛСЭ на фоне основного тона по сравнению с плоским двухчастотным ондулятором с полем (1). Ниже мы проведем моделирование излучения гармоник в ЛСЭ с обычным плоским ондулятором с таким же основным полем, как в (7) и в (1), и сравним результаты для ЛСЭ с обычным плоским, двухчастотным плоским и эллиптическим ондуляторами.

\section{3. ЛСЭ с обычным плоским ондулятором со сдвигом фаз}

Для сравнения с излучением ЛСЭ с двухчастотными ондуляторами мы смоделировали ЛСЭ с обычным плоским ондулятором с тем же числом каскадов и с таким же сдвигом фаз; поле плоского ондулятора получается из (1) с $d=0$ или из (7) с $d_{1}=d_{2}=0$ и соответствует ондулятору в эксперименте LCLS [69,70]; параметры пучка электронов в LCLS также взяты из $[69,70]$. Эволюции банчинга и мощности гармоник излучения по оси ондуляторов показаны соответственно на рис. 9 и 10. В табл. 3 приведены некоторые параметры ЛСЭ, отличные от параметров в табл. 1 и 2. 


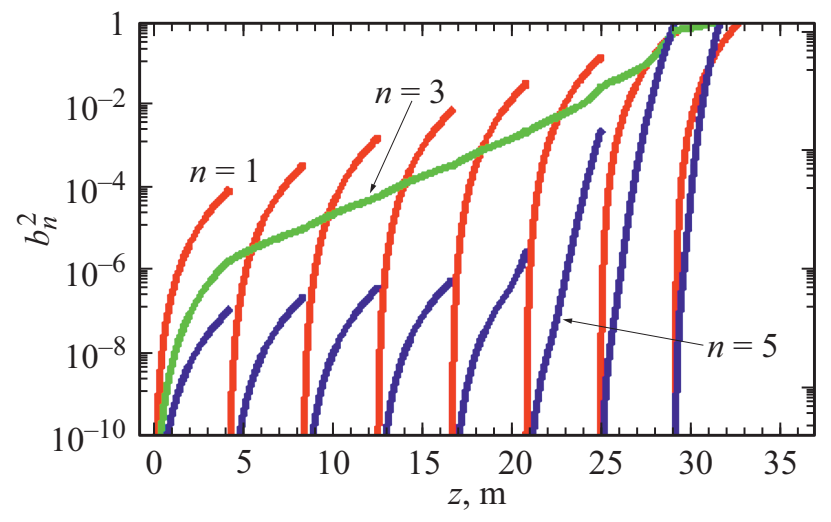

Рис. 9. Коэффициенты банчинга в ЛСЭ со сдвигом фаз между каскадами обычного плоского ондулятора. Номера гармоник $n=1,3,5$ - отмечены на рисунке.

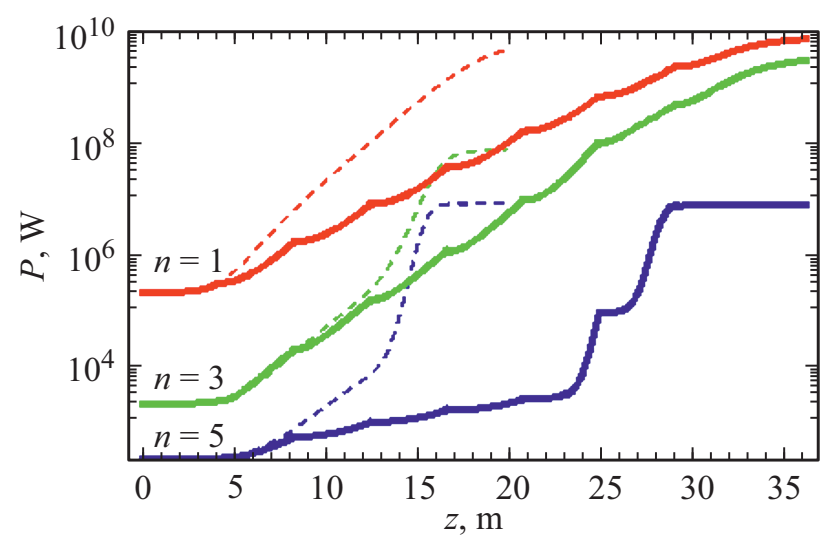

Рис. 10. Мощность гармоник в ЛСЭ со сдвигом фаз между каскадами обычного плоского ондулятора. Номера гармоник $n=1,3,5$ - отмечены на рисунке. Штриховыми линиями обозначены мощности гармоник в ЛСЭ без сдвига фаз между каскадами.

На рис. 9 видно, что происходит разрыв банчинга для $n=1$ и $n=5$, а банчинг для $n=3$ растет непрерывно. Однако ввиду больших значений коэффициентов Бесселя для $n=1$, перегруппировка электронов после сдвига фаз основного тона происходит довольно быстро и мощность 1-й гармоники растет, несмотря на расфазировку. Разрывы банчинга происходят недостаточно часто, чтобы 1-я гармоника оказалась мощнее 3-й в конце ЛСЭ. Сплошные линии на рис. 10 показывают гармоники в ЛСЭ с расфазировкой, тонкие пунктирные - в ЛСЭ без расфазировки. Мощность 3-й гармоники излучения растет в режиме независимой генерации и оказывается выше, чем в ЛСЭ без расфазировки (ср. сплошную и штриховую линии $n=3$ на рис. 10); мощность 5-й гармоники излучения (линия $n=5$ на рис. 9 и 10) растет в нелинейном режиме за счет мощного основного тона (линия $n=1$ на рис. 9 и 10) и достигает значительной мощности насыщения в конце ЛСЭ, несмотря на расфазировку. Чтобы ослабить 1-ю гармонику в ЛСЭ с
Таблица 3. Данные моделирования ЛСЭ с обычным плоским ондулятором

\begin{tabular}{l|c|c|c}
\hline Номер гармоники & $n=1$ & $n=3$ & $n=5$ \\
\hline Коэффициент Бесселя $f_{n ; y}$ & 0.74 & 0.34 & 0.23 \\
\hline Длина усиления гармоники $L_{\text {gain }}, \mathrm{m}$ & 1.5 & 1.9 & 2.2 \\
\hline Параметр Пирса $\rho_{n ; y}$ & 0.0009 & 0.0005 & 0.0004 \\
\hline Длина волны гармоники $\lambda_{n}, \mathrm{~nm}$ & 1.4 & 0.47 & 0.28 \\
\hline $\begin{array}{l}\text { Мощность насыщения гармоник } \\
\text { в ЛСЭ с фазовым сдвигом } P_{F, n}, \mathrm{~W}\end{array}$ & $6 \cdot 10^{9}$ & $2.5 \cdot 10^{9}$ & $9 \cdot 10^{6}$ \\
\hline $\begin{array}{l}\text { Мощность насыщения гармоник } \\
\text { в ЛСЭ без фазового сдвига } P_{F, n}, \mathrm{~W}\end{array}$ & $6 \cdot 10^{9}$ & $7 \cdot 10^{7}$ & $8 \cdot 10^{6}$
\end{tabular}

Примечание. Параметры пучка электронов: см. табл. 1; параметры ондулятора: $k=3.5, \lambda_{u}=2.8 \mathrm{~cm}$; параметры ЛСЭ: параметр Пирса $\tilde{\rho}_{n} \cong(4-9) \cdot 10^{-4}$, длина насыщения для ЛСЭ с фазовым сдвигом $L_{L s 1} \cong 35 \mathrm{~m}, L_{s 3} \cong 30 \mathrm{~m}$, длина насыщения без фазового сдвига $L_{s} \cong 20 \mathrm{~m}$.

обычным ондулятором ниже мощности 3-й гармоники, нужно прерывать ее банчинг примерно на каждой длине усиления.

\section{Выводы}

В настоящей работе, с помощью откалиброванной по экспериментам ЛСЭ феноменологической модели, продемонстрированы преимущества ондуляторов с гармониками магнитного поля для генерации мощных гармоник когерентного излучения. Показано, что с помощью расфазировки электронов и фотонов между секциями ондуляторов можно эффективно ослабить основной тон в ЛСЭ с этими ондуляторами, применяя расфазировку каждые 2-3 длины усиления ЛСЭ; это позволяет повысить мощность насыщения 3-й гармоники излучения до ста раз.

Аналитически получены точные выражения для коэффициентов Бесселя ондуляторов с гармониками магнитного поля; исследовано их поведения в зависимости от напряженности гармоник поля. Показано, что в асимметричном эллиптическом ондуляторе с полем (7), где $h=3, l=1, d_{1}=1, d_{2}=0.2-0.4$, значения коэффициентов Бесселя 1-й, 3-й и 5-й гармоник в $x$-поляризации излучения близки друг другу, $f_{1 ; x} \simeq f_{3 ; x} \simeq f_{5 ; x}$, при этом коэффициент Бесселя основного тона $y$-поляризации $f_{1 ; y}$ имеет наименьшее возможное значение. Для плоского ондулятора с гармоникой поля (1), где $h=3, d=0.5$, 1-я гармоника ОИ ослаблена, а высшие гармоники ОИ усилены. Сделан вывод о том, что ондуляторы с такими магнитными полями могут найти применение в ЛСЭ, в том числе с расфазировкой электронов и фотонов, для генерации мощных гармоник когерентного излучения, превосходящих мощность основного тона.

Проведен сравнительный анализ эволюции коэффициентов группировки и мощности гармоник в ЛСЭ с 
расфазировкой с обычным плоским ондулятором, с двухчастотным плоским ондулятором и с асимметричным эллиптическим ондулятором. Для успешного подавления 1-й гармоники излучения в ЛСЭ обычным ондулятором требуется частый, примерно на каждой длине усиления, сдвиг фаз электронов относительно фотонов. На примере ондулятора LCLS показано, что если расфазировка осуществляется на расстояниях, существенно превышающих длину усиления $L_{g}$, то эффективное подавление основного тона обычного ондулятора ниже мощности 3й гармоники не происходит.

Нами смоделировано излучение ЛСЭ с плоским и эллиптическим ондуляторами с 3-й гармоникой поля и основным полем ондулятора LCLS [69]. Показано, что с параметрами ондулятора $h=3, l=1$, $d_{1}=1, d_{2}=0.2-0.4$ для эллиптического поля (7) или с $d=0.5$ для плоского поля (1) с пучком электронов энергии $E=4.3 \mathrm{GeV}$ и другими параметрами эксперимента LCLS, можно получить 3-ю гармонику излучения на длине волны $\lambda_{3}=0.5-0.6 \mathrm{~nm}$, соответствующей 3-й гармонике LCLS $\lambda_{3, \mathrm{LCLS}}=0.5 \mathrm{~nm}$, с повышенной мощностью $P_{3} \sim 10 \mathrm{GW} \approx P_{1 \text {,LCLS }}$ на длине ондуляторов $\sim L_{s} \approx 35 \mathrm{~m}$. Этого можно достичь с помощью расфазировки электронов и фотонов между секциями ондуляторов длиной $\sim 4 \mathrm{~m}$, т.е. каждые $\sim 2-3$ длины усиления. Мощность излучения на длине волны $\lambda_{3}=0.5-0.6 \mathrm{~nm}$ при этом в сто раз больше, чем в эксперименте LCLS с обычным плоским ондулятором при таких же прочих условиях.

Показано, что в отличие от известных ЛСЭ с обычными ондуляторами в предложенном рентгеновском ЛСЭ с двухчастотным ондулятором 3-я гармоника излучения доминирует после $\sim 30 \mathrm{~m}$ и может индуцировать 3-ю субгармонику на длине волны $\lambda_{3 \times 3}=0.2 \mathrm{~nm} \mathrm{c}$ мощностью $\sim 30 \mathrm{MW}$; это в несколько раз выше мощности пятой гармоники в эксперименте LCLS на более длинной волне, $\lambda_{5}=0.3 \mathrm{~nm}$. Это невозможно в обычном плоском ондуляторе из-за малости коэффициентов Бесселя и параметра Пирса для высших гармоник.

Таким образом, нами показано, что использование в ЛСЭ ондуляторов с 3-й гармоникой магнитного поля позволяет значительно повысить максимальную мощность 3-й гармоники излучения в рентгеновском диапазоне. Применяя в ЛСЭ с двухчастотными ондуляторами расфазировку электронов и фотонов каждые 2-3 длины усиления, можно повысить мощность насыщения третьей гармоники излучения в сто раз до мощности основного тона. При этом становится возможной генерация субгармоник доминирующей 3-й гармоники ЛСЭ на длинах волн менее нанометра. В ЛСЭ с обычным плоским ондулятором для подавления основного тона требуется значительно более частый фазовый сдвиг электронов относительно фотонов.

В предложенных ЛСЭ с двухчастотными ондуляторами в отличие от существующих установок осуществляется генерация мощных гармоник рентгеновского излу- чения с меньшей энергией электронов и, следовательно, требуются меньшие размеры и стоимость установки.

\section{Благодарности}

Авторы выражают благодарность проф. А. Борисову и гл.н.с. А. Лобанову за полезные обсуждения и советы. Один из авторов (А.М.К.) выражает благодарность Фонду развития теоретической физики и математики „Базис“ за оказанную поддержку

\section{Конфликт интересов}

Авторы заявляют, что у них нет конфликта интересов.

\section{Список литературы}

[1] Багров В.Г., Бисноватый-Коган Г.С., Бордовицын В.А., Борисов А.В., Дорофеев О.Ф., Эрр В.Я., Пивоваров Ю.Л., Шорохов О.В., Жуковский В.Ч. Теория излучения релятивистских частиц / Под ред. В.А Бордовицина. М.: Физматлит, 2002.

[2] Гинзбург В.Л. // Изв. АН СССР. Сер. Физ. 1947. Т. 11. № 2 C. 165-181. [Ginzburg V.L. Isvestia Akademii Nauk SSSR (Fizika). 1947. Vol. 11. N 2. P. 1651.

[3] Motz H., Thon W., Whitehurst R.N.J. // Appl. Phys. 1953. Vol. 24. P. 826.

[4] McNeil B.W.J., Thompson N.R. // Nature Photonics. 2010. Vol. 4. P. 814.

[5] Pellegrini C., Marinelli A., Reiche S. // Rev. Mod. Phys. 2016. Vol. 88. P. 015006.

[6] Huang Z., Kim K.J. // Phys. Rev. ST-AB. 2007. Vol. 10. P. 034801.

[7] Saldin E.L., Schneidmiller E.A., Yurkov M.V. The Physics of Free Electron Lasers. Springer, 2000.

[8] Bonifacio R., Pellegrini C., Narducci L. // Opt. Commun. 1984. Vol. 50. P. 373. DOI: $10.1016 / 0030-4018(84) 90105-6$

[9] Schmüser P., Dohlus M., Rossbach J., Behrens C. // Springer Tracts in Modern Phys. 2004. Vol. 258.

[10] Pellegrini C. // Phys. Scr. 2016. Vol. 2016. P. 014004.

[11] Margaritondo G., Ribic P.R. // J. Synchrotron Rad. 2011. Vol. 18. P. 101-108.

[12] Margaritondo G. // Rivista del Nuovo Cimento. 2017. Vol. 40. N 9. P. 411-471.

[13] Margaritondo G. Characteristics and properties of synchrotron radiation, in Synchrotron Radiation: Basics, Methods and Applications. 2015. P. 29-63.

[14] Albertin F., Astolfo A., Stampanoni M., Kaplan F., Margaritondo G. // X-Ray Spectrometry. 2015. Vol. 44. N 3. P. 93-98.

[15] Frank M., Carlson D.B., Hunter M.S., Williams G.J., Messerschmidt M., Zatsepin N.A., Barty A., Benner W.H., Chu K., Graf A.T., Hau-Riege S.P., Kirian R.A., Padeste C., Pardini T., Pedrini B., Segelke B., Seibert M.M., Spence J.C.H., Tsai C.-J., Lane S.M., Li X.-D., Schertler G., Boutet S., Coleman M., Evans J.E. Femtosecond X-ray diffraction from two-dimensional protein crystals, IUCrJ, 2014. Vol. 1. P. 95.

[16] Margaritondo G. // J. Synchrotron Rad. 2019. Vol. 26. P. 2094-2096 
[17] Hwu Y., Margaritondo G., Chiang A.-S. // BMC Biology. 2017. Vol. 15. P. 122

[18] Huang C.-F., Liang K.S., Hsu T.-L., Lee T.-T., Chen Y.-Y., Yang S.-M., Chen H.-H., Huang S.-H., Chang W.-H., Lee T.K., Chen P., Peng K.-E., Chen C.-C., Shi C.-Z., Hu Y.F., Margaritondo G., Ishikawa T., Wong C.-H., Hwu Y. // Nanoscale. 2018. Vol. 10. P. 2820-2824.

[19] Kroll N.M., McMullin W.A. // Phys. Rev. A. 1978. Vol. 17. N 1. P. 300.

[20] Colson W.B. // Nucl. Instrum. Meth. A. 1997. Vol. 393. P. $82-$ 85.

[21] Sprangle P., Smith R.A. // Phys. Rev. A. 1980. Vol. 21. N 1. P. 293.

[22] Bonifacio R., Pellegrini C., Narducci L.M. // Opt. Commun. 1984. Vol. 50. P. 373-378.

[23] Kim K.J., Xie M. // Nucl. Instrum. A. 1993. Vol. 331. P. 359 364.

[24] Yu L.-H., Babzien M., Ben-Zvi I., DiMauro L.F., Doyuran A., Graves W., Johnson E., Krinsky S., Malone R., Pogorelsky I., Skaritka J., Rakowsky G., Solomon L., Wang X.J., Woodle M., Yakimenko V., Biedron S.G., Galayda J.N., Gluskin E., Jagger J., Sajaev V., Vasserman I. // Science. 2000. Vol. 289. P. 932.

[25] Yu L.-H. // Phys. Rev. A. 1991. Vol. 44. P. 5178.

[26] Saldin E.L., Schneidmiller E.A., Yurkov M.V. // Opt. Commun. 2002. Vol. 202. P. 169-187.

[27] Shaftan T., Yu L.-H. // Phys. Rev. E. 2005. Vol. 71. P. 046501.

[28] Li He-Ting, Jia Qi-Ka // Chinese Phys. C. 2013. Vol. 37. N 2. P. 028102

[29] Deng Hai-Xiao, Dai Zhi-Min. // Chinese Phys. C. 2013. Vol. 37. N 10. P. 102001.

[30] Deng Hai-Xiao, Dai Zhi-Min. // Chinese Phys. C. 2010. Vol. 34. N 8. P. 1140.

[31] Zeng L., Qin W., Zhao G., Huang S., Ding Y., Huang Zh., Marcus G., Liu K. // Chinese Phys. C. 2016. Vol. 40. N 9. P. 098102.

[32] Yu L.H., DiMauro L., Doyuran A., Graves W.S., Johnson E.D., Heese R., Krinsky S., Loos H., Murphy J.B., Rakowsky G., Rose J., Shaftan T., Sheehy B., Skaritka J., Wang X.J., Wu Z. // Phys. Rev. Lett. 2003. Vol. 91. P. 074801.

[33] McNeil B. // Nature Photon. 2008. Vol. 2. P. 522-524. DOI: $10.1038 /$ nphoton.2008.164

[34] Tiedtke K., Azima A., von Bargen N., Bittner L., Bonfigt S., Düsterer S., Faatz B., Frühling U., Gensch M., Gerth Ch., Guerassimova N., Hahn U., Hans T., Hesse M., Honkavaar K., Jastrow U., Juranic P., Kapitzki S., Keitel B., Kracht T., Kuhlmann M., Li W.B., Martins M., Núñez T., Plönjes E., Redlin H., Saldin E.L., Schneidmiller E.A., Schneider J.R., Schreiber S., Stojanovic N., Tavella F., Toleikis S., Treusch R., Weigelt H., Wellhöfer M., Wabnitz H, Yurkov M.V., Feldhaus J. // New J. Phys. 2009. Vol. 11. P. 023029.

[35] Жуковский К.В. // Вестн. Моск. ун-та. Сер. 3. Физ. Астрон. 2015. T. 4. C. 18. [Zhukovsky K.V. // Moscow Univ. Phys. Bull. 2015. Vol. 70. P. 232.]

[36] Zhukovsky K. // Laser Part. Beams. 2016. Vol. 34. P. 447.

[37] Mishra G., Gehlot M., Hussain J.-K. // Nucl. Instrum. A. 2009. Vol. 603. P. 495.

[38] Zhukovsky K.V. // J. Synchrotron Rad. 2019. Vol. 26. P. 14811488.

[39] Henderson J.R., Campbell L.T., Freund H.P., McNeil B.W.J. // New J. Phys. 2016. Vol. 18. P. 062003.
[40] Freund H.P., van der Slot P.J.M., Grimminck D.L.A.G., Setija I.D., Falgari P. // New J. Phys. 2017. Vol. 19. P. 023020

[41] Freund H.P., van der Slot P.J.M. // New J. Phys. 2018. Vol. 20. P. 073017.

[42] Жуковский К.В. // Изв. вузов. Физика. 2019. Т. 62. № 6. C. 109-117 [Zhukovsky K.V. // Russ. Phys. J. 2019. Vol. 62 N 6. P. 1043-1053].

[43] Zhukovsky K. // Results in Physics. 2019. Vol. 13. P. 102248.

[44] Alexeev V.I., Bessonov E.G. // Nucl. Instr. Meth. 1991. Vol. A308. P. 140.

[45] Dattoli G., Mikhailin V.V., Ottaviani P.L., Zhukovsky K. // J. Appl. Phys. 2006. Vol. 100. P. 084507.

[46] Dattoli G., Mirian N.S., DiPalma E., Petrillo V. // Phys. Rev. ST-AB. 2014. Vol. 17. P. 050702.

[47] Zhukovsky K., Potapov I. // Laser Part. Beams. 2017. Vol. 35. P. 326.

[48] Жуковский К.В. // Известия вузов. Физика. 2017. Т. 60 № 9. C. 155-161 [Zhukovsky K.V. // Russ. Phys. J. 2018. Vol. 60. N 9. P. 1630-1637].

[49] Zhukovsky K., Kalitenko A. // J. Synchrotron Rad. 2019. Vol. 26. P. 159-169.

[50] Жуковский К.В. Калитенко А.М. // Изв. вузов. Физика. 2019. T. 62. № 2. C. 153-160 [Zhukovsky K.V., Kalitenko A. // Russ. Phys. J. 2019. Vol. 62. N 2. P. 354-362].

[51] Zhukovsky K., Kalitenko A. // J. Synchrotron Rad. 2019. Vol. 26. P. 605-606.

[52] Zhukovsky K. // J. Phys. D. 2017. Vol. 50. P. 505601.

[53] Zhukovsky K. // EPL. 2017. Vol. 119. P. 34002.

[54] Жуковский К.В. // ЖТФ. 2019. Т. 89. Вып. 3. С. 426-435. [Zhukovskii K.V. // Tech. Phys. 2019. Vol. 64. N 3. P. 389 398.]

[55] Жуковский К.В. Потапов И.А., Калитенко А.М. // Изв. вузов. Радиофизика. 2018. Т. 61. № 3. С. 244-260 [Zhukovsky K.V., Potapov I.A., Kalitenko A.M. // Radiophys. Quant. El. 2018. Vol. 61 N 3. P. 216-231].

[56] Жуковский К.В. // Вестн. Моск. ун-та. Сер. 3. Физ. Астрон. 2018. Вып. 4. С. 26-34 [Zhukovsky K.V. // Moscow Univ. Phys. Bull. 2018. Vol. 73. N 4. P. 364-371].

[57] Zhukovsky K. // Opt. Commun. 2018. Vol. 418. P. 57-64.

[58] Zhukovsky K. // J. Appl. Phys. 2017. Vol. 122. P. 233103.

[59] Schneidmiller E.A., Yurkov M.V. // Phys. Rev. ST-AB. 2012. Vol. 15. P. 080702.

[60] McNeil B.W.J., Robb G.R.M., Poole M.W., Thompson N.R. // Phys. Rev. Lett. 2006. Vol. 96. P. 084801.

[61] Жуковский К.В. // Вестн. Моск. ун-та. Сер. 3. Физ. Астрон. 2018. Вып. 5. C. 18-25 [Zhukovsky K.V. // Moscow Univ. Phys. Bull. 2018. Vol. 73. N 5. P. 462-469].

[62] Zhukovsky K. // J. Optics. 2018. Vol. 20. N 9. P. 095003.

[63] Zhukovsky K.V. // Moscow Univ. Phys. Bull. 2019. Vol. 74. N 3. P. $308-308$.

[64] Жуковский К.В. // Вестн. Моск. ун-та. Сер. 3. Физ. Астрон. 2019. Вып. 5. С. 60-66 [Zhukovsky K.V. // Moscow Univ. Phys. Bull. 2019. Vol. 74. N 5. P. 480-487].

[65] Zhukovsky K. // Nucl. Instrum. B. 2016. Vol. 369. P. 9.

[66] Zhukovsky K. // Opt. Commun. 2015. Vol. 353. P. 35.

[67] Bonifacio R., De Salvo L., Pierini P. // Nucl. Instrum. A. 1990. Vol. 293. P. 627.

[68] Huang Z., Kim K.-J. // Phys. Rev. E. 2000. Vol. 62. P. 7295. 
[69] Emma P., Akre R., Arthur J., Bionta R., Bostedt C., Bozek J., Brachmann A., Bucksbaum P., Coffee R., Decker F.J., Ding Y., Dowell D., Edstrom S., Fisher A., Frisch J., Gilevich S., Hastings J., Hays G., Hering Ph., Huang Z., Iverson R., Loos H., Messerschmidt M., Miahnahri A., Moeller S., Nuhn H.-D., Pile G., Ratner D., Rzepiela J., Schultz D., Smith T., Stefan P., Tompkins H., Turner J., Welch J., White W., Wu J., Yocky G., Galayda J. // Nature, Photonics. 2010. Vol. 4. P. 641-647.

[70] Ratner D., Brachmann A., Decker F.J., Ding Y., Dowell D., Emma P., Fisher A., Frisch J., Gilevich S., Huang Z., Hering P., Iverson R., Krzywinski J., Loos H., Messerschmidt M., Nuhn H.D., Smith T., Turner J., Welch J., White $W$., $W u$ J. // Phys. Rev. ST-AB. 2011. Vol. 14. P. 060701. 